\title{
Solution of coupled nonlinear Schrödinger equations in a focusing- defocusing medium by modified perturbation theory
}

\author{
Jerzy Ja siński, Mirosław Ka rpierz \\ Facultyof Physics, Warsaw University of Technology, Koszykowa 75, 00-662 Warszawa
}

Received June 06, 2021; accepted June 28, 2021; published June 30, 2021

\begin{abstract}
Interaction is considered of bright solitons of different orders and two different wavelengths propagating in a medium focusing for one wavelength and defocusing for the other. The system of nonlinear Schrödinger equations is solved by means of perturbation theory. The application of an additional postulate to adjust both widths of the solitons and to modify the amplitude by a factor determined by the overlap integral greatly improves the accuracy of the description. Good accuracy of description is confirmed by numerical calculations.
\end{abstract}

In nonlinear optics, the coupled nonlinear Schrö dinger equations have been for many years the main tool for studying the interactions of solitons with each other and with the medium through which they pass [1-6]. In this paper, we consider a nonlinear medium focusing for a wave at one frequency and defocusing for another and the description of interaction between two such wa ves.

Consider two beams $U_{P o s}(x, z)$ and $U_{N e g}(x, z)$ interacting with a nonlinear medium of nonlinearity:

$$
\varepsilon_{2}=\alpha_{P}\left|U_{P o s}\right|^{2}-\alpha_{N}\left|U_{N e g}\right|^{2},
$$

for $\alpha_{P}>\alpha_{N} \geq 0$. The Nonlinear Schrödinger Equations (NSE) describing the propagation of beams have the form:

$$
\begin{aligned}
& i \beta_{P} \frac{\partial U_{P o s}}{\partial z}+\frac{1}{2} \frac{\partial^{2} U_{P o s}}{\partial x^{2}}+\left(\alpha_{P}\left|U_{P o s}\right|^{2}-\alpha_{N}\left|U_{N e g}\right|^{2}\right) U_{P o s}=0 \\
& i \beta_{N} \frac{\partial U_{N e g}}{\partial z}+\frac{1}{2} \frac{\partial^{2} U_{N e g}}{\partial x^{2}}+\left(\alpha_{N}\left|U_{P o s}\right|^{2}-\alpha_{N}\left|U_{N e g}\right|^{2}\right) U_{N e g}=0 .
\end{aligned}
$$

This case wa s considered by many authors [4, 6-10] and discussed using different attitudes - analytical [1, 4, 7, 9], numerical [10] or varia tional $[6,8]$.

But the simplest solution of Eqs. (2) describes the case of vanishing field $U_{\text {Neg }}$. Normalizing the wa ve function $U_{P o s}$ together with the coordinates $(x, z)$ gives NSE in its fundamental form:

$$
i \frac{\partial \Psi_{n}}{\partial z}+\frac{1}{2} \frac{\partial^{2} \Psi_{n}}{\partial x^{2}}+\left|\Psi_{n}\right|^{2} \Psi_{n}=0 .
$$

The series of solutions of Eq. (3) can be obtained via Inverse Scattering Transform (IST) [4]. They represent solitons of different orders $n=1,2, \ldots$ The function $\Psi_{n}$ is a quotient of two complex combinations of $n$ term s of the form $\exp \left(-2 \eta_{l}\left(x-x_{0 l}\right)+2 i \eta_{l}^{2}\left(z-z_{0 l}\right)\right)$ with arbitrary real $\eta_{l}$, $x_{0 l}$, and $z_{0 l}(l=1, . . n)$. The coefficients $\eta_{l}$ determine the widths and heights of its individual components.
A system of equations like Eqs. (2) for $\beta_{P}=\beta_{N}=1$ and focusing nonlinearity for both beams $\alpha_{P}=1, \alpha_{N}=-1$ h a s an analytical solution known as Manakov solitons [4, 10-14]. Based on Manakov's solution, an analogous solution of the system [Eqs. (2)] for focusing-defocusing nonlinearity has been discussed in [4]. Both fields $U_{P o s}$ and $U_{N e g}$ are described by solitons of the same order, but unfortunately this solution exists only for the special ca se where $\alpha_{P}=\alpha_{N}=1$ and $\beta_{P}=\beta_{N}=1$.

The con sidered system of Eqs. (2) generalizes this case. To obta in Manakov-type solution we should in troduce a dditional amplitude factors $\gamma_{P o s}$ and $\gamma_{N e g}$ :

$$
\begin{aligned}
& U_{\text {Pos }}=\gamma_{P o S} \Psi_{n}, \\
& U_{N e g}=\gamma_{N e g} \Psi_{n} .
\end{aligned}
$$

Assume the real $\gamma_{P o s}$ and $\gamma_{\text {Neg }}$ (their phases are included into initial phase factors of $\Psi_{n}$ ). Substituting Eqs. (4) in to Eqs. (2) we can prove that Eq. (3) is satisfied only for:

$$
\begin{gathered}
\beta_{P}=\beta_{N}=1, \\
\alpha_{P} \gamma_{p o s}^{2}-\alpha_{N} \gamma_{N e g}^{2}=1 .
\end{gathered}
$$

The requirement (4) implies that solitons of the both fields $U_{P o s}$ and $U_{N e g}$ have not only the same order $n$, but the centra $x_{0 l}$ of all their corresponding components for $l_{\text {Pos }}=l_{\text {Neg }}=1, . . n$ have the same positions. In addition, for any pair of component indices $l_{1}$ and $l_{2}$ the initial phase differences $\eta_{l 1}^{2} z_{0 l 1}-\eta_{l 2}^{2} z_{0 l 2}$ are the same for both fields.

In this paperwe solve the system of Eqs. (2) a pply ing perturbation theory. This attitude is frequently applied to describe various effects connected with interaction between solitons or with the medium $[1,5]$. Although the $U_{N e g}$ field will not a lways be small relative to $U_{P o s}$, let u s treat it initially as a small perturbation. Expressing both fields a series with respect to a small constant quantity $o$ of order of magnitude $U_{N e g}$ we have:

$$
\begin{aligned}
& U_{P o s}=U_{P}^{(0)}+o U_{P}^{(1)}+o^{2} U_{P}^{(2)}+\ldots, \\
& U_{\text {Neg }}=o U_{N}^{(1)}+\ldots
\end{aligned}
$$

The unperturbed field $U_{P}{ }^{(0)}$ satisfies thefirst equation of the system (2) with vanishing $U_{N e g}$. Its solution describes a soliton of a rbitrary order $n$ :

$$
U_{P}^{(0)}(x, z)=\gamma_{P}^{(0)} \Psi_{n}(x, z),
$$


with $\gamma_{P}{ }^{(0)}$ denoting the same symbol as in Eqs. (4) and (5), but written for vanishing $U_{N e g}$. For $n=1$ this solution gives:

$$
U_{P}^{(0)}=\gamma_{P}^{(0)} \Psi_{1}=A_{P} e^{i\left(k_{P} z+\varphi_{0 P}\right)} \operatorname{Sech}\left(2 \eta_{P} x\right),
$$

with $k_{P}=2 \eta_{P}^{2} / \beta_{P} \quad$ and $=2 \eta_{P} / \sqrt{\alpha_{P}}=2 \eta_{P} \gamma_{P}^{(0)}$. The initial phase $\varphi_{0 P}$ is a rbitrary.

By substituting the expansion (6) into the system (2), we prove that the first-order correction vanishes $U_{P}^{(1)} \equiv 0$ while $U_{N}^{(1)}$ satisfies the equation:

$$
i \beta_{N} \frac{\partial U_{N}^{(1)}}{\partial z}+\frac{1}{2} \frac{\partial^{2} U_{N}^{(1)}}{\partial x^{2}}+\alpha_{N}\left|U_{P}^{(0)}\right|^{2} U_{N}^{(1)}=0 .
$$

The equation for the Neg field $U_{N}^{(1)}$ is linear. Its solution gives a function with a profile like $U_{P}{ }^{(0)}$, but it may havea different propagating term:

$$
U_{N}^{(1)}(x, z)=a_{N} e^{i \Delta k z} U_{P}^{(0)}(x, z),
$$

Nevertheless, substituting (10)into Eq. (9), we prove that it is satisfied only for two cases: 1) $\beta_{N}=\beta_{P}, \Delta k=0$ or 2 ) $U_{P}^{(0)}=\Psi_{1}$ and $\Delta k=2 \eta_{P}^{2}\left(1 / \beta_{1}-1 / \beta_{P}\right)$. Of particular interest is the second case, in which two solitons $U_{P o s}$ and $U_{N e g}$ can correspond to two different wa velengths.

But one can also consider solutions of Eq. (9), where instead of a true distribution of the refractive index derived from the nonlinearity $\alpha_{N}\left|U_{P}{ }^{(0)}\right|^{2}$ we substitute a distribution that is close, but slightly different. Assume that this slightly different distribution is given by:

$$
\left|U_{P}^{(0)}(x, z)\right|^{2} \approx U_{d}^{2}=A_{d}^{2} \operatorname{Sech}^{2}\left(2 \eta_{d} x\right)
$$

The parameter $\eta_{d}$ can slightly differ from one of the numbers $\eta_{P 1} \ldots \eta_{P n}$, defining $n$-th order soliton (7), but the relation between $A_{d}$ and $\eta_{d}$ are assumed the same a s the first-order soliton: $A_{d}=2 \eta_{d} / \sqrt{\alpha_{P}}$. Moreover, to minimize deviation from the $z$-axis during propagation, as sume a $n$ approximately symmetrical shape of the soliton $\left|U_{P}^{(0)}(x, z)\right|$ in the whole range of propagation.

The approximation (11) gives the solution of Eq. (9):

$$
U_{N}^{(1)}=\gamma_{N}^{(1)} \sqrt{\alpha_{P}} U_{d}=A_{N} e^{i k_{N} z} \operatorname{Sech}\left(2 \eta_{d} x\right),
$$

with $k_{N}=2 \eta_{P d}^{2} / \beta_{N}$ but arbitrary a mplitude $\gamma_{N}^{(1)}$ (or $A_{N}$ ) of $\underline{U}_{N e g}$ field (the multiplier before $U_{d}$ is for consistency with designations in expressions (4)).

The first non-vanishing correction $U_{P}^{(2)}$ for $U_{P o s}$ field satisfies a much more complicated equation:

$$
\begin{gathered}
i \beta_{P} \frac{\partial U_{P}^{(2)}}{\partial z}+\frac{1}{2} \frac{\partial^{2} U_{P}^{(2)}}{\partial x^{2}}+\alpha_{P o s}\left(2\left|U_{P}^{(0)}\right|^{2} U_{P}^{(2)}+\left(U_{P}^{(0)}\right)^{2} U_{P}^{*(2)}\right) \\
=\alpha_{\text {Neg }}\left|U_{N e g}^{(1)}\right|^{2} U_{P}^{(2)}
\end{gathered}
$$

Nevertheless, assuming $U_{N}^{(1)}$ of the form (12) and:

$$
U_{P}^{(2)}=\gamma_{P}^{(2)} \sqrt{\alpha_{P}} U_{P}^{(0)}
$$

we a re able to find the solution of Eq. (13) for real $\gamma_{N}{ }^{(1)}$ and $\gamma_{P}{ }^{(2)}$. Substituting (12) and (14) into Eq. (13) gives the condition for the ex istence of this solution:

$$
\gamma_{P}^{(2)}=\alpha_{\text {Neg }} \frac{\left(\gamma_{N}^{(1)}\right)^{2}}{2 \alpha_{\text {Pos }} \gamma_{P}^{(0)}} .
$$

The obtained perturbed solutions of Eqs. (7)-(15) contain at least one case of different propagation constants $\beta_{N} \neq \beta_{P}$, but also cover the case of Manakovtype solution, Eqs. (4)-(5). To compare results following from these two attitudes, we should assume equal $\beta_{N}$ and $\beta_{P}$, as in Eq. (5). Now we can see that treating $\gamma_{N e g} \approx o \gamma_{N}^{(1)}$ a s a small quantity we have:

$$
\gamma_{P o s}=\sqrt{\frac{1+\alpha_{N} \gamma_{N e g}^{2}}{\alpha_{P}}} \approx \frac{1}{\sqrt{\alpha_{P}}}+o^{2} \frac{\alpha_{N}\left(\gamma_{N}^{(1)}\right)^{2}}{2 \sqrt{\alpha_{P}}}=\gamma_{P}^{(0)}+o^{2} \gamma_{P}^{(2)} .
$$

Since the obtained second-order correction in expansion (16) is identicalas (15), the conclusions from the exact Manakov-type solution (4)-(5) and the perturbation theory (7)-(15) are the samefor the samechoice of parameters. But the perturbation theory gives more possibilities to change the parameters of the interacting solitons.

By now the amplitude term $\gamma_{N}{ }^{(1)}$ is a rbitrary, because Eq. (9) is linear. To establish this term let us define the overla p integral $Q$ ca lculated using the initial field shapes:

$$
Q=\frac{\int_{-\infty}^{\infty}\left|U_{P}(x, 0)\right| \cdot\left|U_{N}(x, 0)\right| d x}{\sqrt{\int_{-\infty}^{\infty}\left|U_{P}(x, 0)\right|^{2} d x \cdot \int_{-\infty}^{\infty}\left|U_{N}(x, 0)\right|^{2} d x}} .
$$

Of course, $0 \leq Q \leq 1$. During propagation the Neg soliton and central peak of the Pos soliton equal their widths. The amplitude of the $U_{N e g}$ field will decrea se or increase, depending on the relation between $\eta_{N}$ and $\eta_{P}$. But its power a lwa ys decreases to $Q^{2}$ of the initial power ( $U_{\text {Neg }}$ field is much smaller than $U_{\text {Pos }}$, so the power carried by Pos soliton hardly changes). This gives the rule ena bling us to determine the amplitude of $U_{N e g}$ field:

$$
U_{Q}=\lim _{z \rightarrow \infty}\left|U_{\text {Neg }}(0, z)\right|=Q \cdot \sqrt{\frac{\eta_{d}}{\eta_{N}}} \cdot\left|U_{\text {Neg }}(0,0)\right| .
$$

Of course, both solitons should retain their en ergy, as proved in [9]. But in the process of adjusting their width $\mathrm{s}$ some of the wa ves quickly escape outside the re gion of interaction. Thus, the reduction in power applies only to the fields that remain in the system still intera c ting with each other.

To check the obtained results numerically we a s sumed $\alpha_{P}=1, \alpha_{N}=0.25, \beta_{P}=1$ and $\beta_{N}=0.8$. As the input $U_{P o s}$ bea $\mathrm{m}$ we took a third-order soliton with the central peak corresponding to $\eta_{P}=3$ at $z$-axis $\left(x_{P 10}=0\right)$ and two side peaks approximately twice higher than the central peak. $U_{N e g}$ beam at the input contained the first-ord er solito $n$ defined by parameter $\eta_{N}=10$ with a mplitude $2 / 3$ height of the central Pos peak. The other initial parameters have 
been adjusted to obtain the side peaks clearly separated from the central maximum. For these values in the in put plane on the $x$-axis we have $\alpha_{P}\left|U_{P o s}\right|^{2} \approx 0.7 \alpha_{N}\left|U_{N e g}\right|^{2}$, which means that at the beginning of propagation the $U_{N e g}$ beam reduces the nonlinear susceptibility over 3 times, so the intera ction of solitons cannot be considered weak.
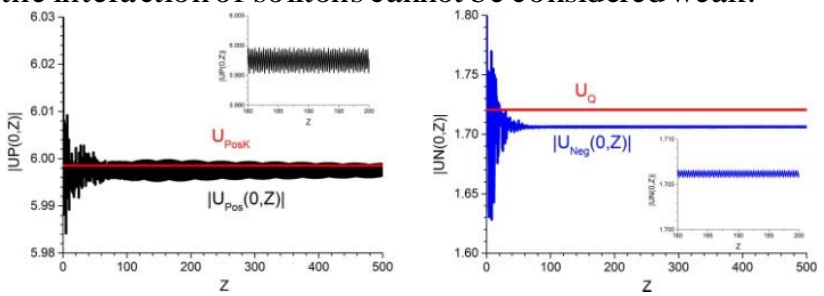

Fig. 1. Central heights of $\mathrm{U}_{P o s}$ and $U_{\text {Neg }}$ fields during propagation over the distance $z_{K}=500$. $U_{\text {Pos }}(0,0)=6.03, U_{\text {Neg }}(0,0)=1.8$.

In Fig. 1 we can see how the central heights of both solitons change during propagation. Note that the heigh ts of the two fields initially decrease, but after a distance of about 70, fairly regula roscilla tions of both a mplitudes remain in the system with a small rela tive amplitu de of $3.4 \cdot 10^{-4}$ for $U_{P o s}$ and 5 times less for $U_{N e g}$. Moreover, we can see that the amplitude of the Neg soliton changes rapidly at the very initial stage of propagation.
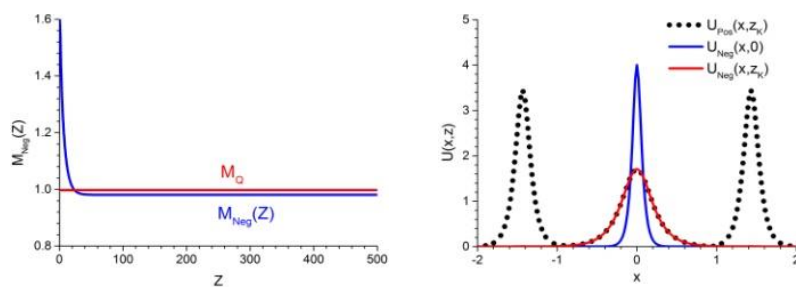

Fig. 2. The change of power of the propagating Neg field (left) and comparison of the initial and final profiles (right).

Ana logous behaviour can be seen for the power $M_{N e g}=$ $\int_{-\infty}^{\infty}\left|U_{N}(x, z)\right|^{2} d x$ of the Neg soliton (left graph of Fig. 2). On the other hand, in the right graph one can observe that the profile of the $\mathrm{Neg}$ field and the central peak of the Pos field a gree very well (the Pos field is scaled to obtain the same central heights). Adjusting Sech function to the final profile of both beams, we find $\eta_{d}=2.9686$, which is $1 \%$ less than the assumed value $\eta_{P}=3$ for the initia 1 central peak. For the initial shapes of both considered solitons, we obtain $Q=0.7894$, which gives the estimated value of power with a fairly good $1 \%$ a ccuracy (the red line). Using the obtained $\eta_{d}$, one can calculate $\gamma_{N}^{(1)}=0.2874$ and $\gamma_{P}{ }^{(2)}$ $=0.0103$, which gives the final height of the Neg solit on drawn by the red line in the right graph of Fig. 1 (accuracy $0.2 \%$ nevertheless is a significant change) and the final height of the Pos soliton drawn by the black line in the left graph (accuracy $0.01 \%$ ).

Of course, the accuracy of the method increases for closer values of the central widths (closer $\eta_{P}$ and $\eta_{N}$ ). But it is also important to have a clearly marked peak in the centre of the Pos soliton field distribution. In Fig. 3 we show the final fields for the initial central peak height equal0.3 (the left graph) and 0.25 (the right graph) of the side peaks. All other parameters are the same. In the left graph one can hardly distinguish deformations (howe ver both final fields lost their symmetry), but in the right graph we can see that propagation became unstable - the Neg field is no longer guided by Pos soliton.
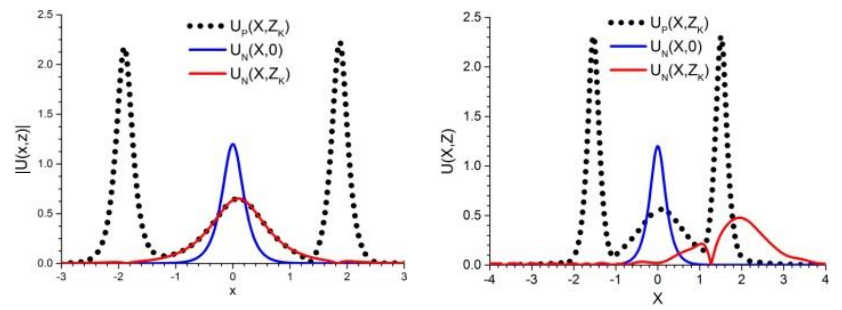

Fig. 3. Deformations of the final profiles of Neg solitons.

In conclusions, using the perturbation the ory we have found solutions in the form of a pair of solit a ry beams with different orders, propagating in the focusingdefocusing medium. The proposed method gives very good quantitative results and calculated beams a re st ab le at long dista nces. Simila r multi-hump solitons have been reported recently, however, in a nonlocal nonlinear medium [13-15].

This work is supported by the Polish National Science Center through project 2016/22/M/ST2/00261

\section{References}

[1] Y. Kivshar, G. P. Agrawal, Optical Solitons. From Fibers to Photon ic Crystals, (Amsterdam, Academic Press 2003).

[2] F. Abdullaev, S. Darmanyan, P. Khabibullaev, Optical Solitons, (Springer-Verlag, Berlin, 1993).

[3] G.I.A. Stegeman, D.N. Christodoulides, M. Segev, IEEE J. S elected Topics Quantum Electron. 6, 1419(2000).

[4] J. Yang, Nonlinear Waves in Integrable and Nonintegrable Systems, (SIAM, Philadelphia 2010).

[5] Y. Kivshar, B. Malomed, Rev. Mod. Phys. 61, 763 (1989).

[6] P.G. Kevrekidis, D.J. Frantzeskakis, Reviews in Physics 1, 140 (2016).

[7] R. de la Fuente, A. Barthelemy, IEEE J. Quantum Electron. 28, 547 (1992).

[8] H.T. Tran, R.A. Sammut, Phys. Rev. A 52,3170(1995).

[9] S. Leble, B. Reichel, Eur. Phys. J. Special Topics 173, 5 (2009).

[10] M. Vijayajayanthi, T.Kanna, M. Lakshmanan, Eur. Phys. J. S pecial Topics 173, 57 (2009).

[11] S. V. Manakov, Sov. Phys. JETP 38(1973), 248

[12] J. Yang, Phys. Rev. E 65, 036606 (2002).

[13] T.Kanna, M. Lakshmanan, Phys. Rev. Lett. 86, 5043(2001).

[14] M. Jakubowski, K. Steiglitz, R. Squier, Phys. Rev. E 58, 6752(1998).

[15] P.S. Jung, W. Krolikowski, U.A. Laudyn, M. Trippenbach, M.A. Karpierz, Phys. Rev. A 95 (2017).

[16] P.S. Jung, M.A. Karpierz, M. Trippenbach, D.N. Chri st od oulides, W. Krolikowski, Photon. Lett. Poland 10(2018).

[17] A. Ramaniuk, M. Trippenbach, P.S. Jung, D.N. Christodoulides, W.Krolikowski, G. Assanto, Opt. Express 29, 8015 (2021). 